\title{
Common Fixed Point Theorems for Subcompatible $D$-Maps
}

\author{
H. Bouhadjera, A. Djoudi, And Brian Fisher
}

\begin{abstract}
The purpose of this paper is to establish a common fixed point theorem for two pairs of subcompatible single and set-valued $D$ maps in a metric space. This result improves, extends and generalizes the result of [1] and others.
\end{abstract}

\section{INTRODUCTION}

In the sequel $(\mathcal{X}, d)$ denotes a metric space and $B(\mathcal{X})$ is the set of all nonempty bounded subsets of $\mathcal{X}$. We define

$$
\delta(A, B)=\sup \{d(a, b): a \in A, b \in B\}
$$

for all $A, B$ in $B(\mathcal{X})$. If $A=\{a\}$, we write $\delta(A, B)=\delta(a, B)$. Also, if $B=\{b\}$, we write $\delta(A, B)=d(a, b)$. From the definition of $\delta(A, B)$ it follows immediately that

$$
\begin{aligned}
& \delta(A, B) \geq 0, \\
& \delta(A, B)=\delta(B, A), \\
& \delta(A, B) \leq \delta(A, C)+\delta(C, B), \\
& \delta(A, A)=\operatorname{diam} A, \\
& \delta(A, B)=0 \quad \text { iff } A=B=\{a\}
\end{aligned}
$$

for all $A, B, C$ in $B(\mathcal{X})$.

Definition $1.1([3])$. A sequence $\left\{A_{n}\right\}$ of nonempty subsets of $\mathcal{X}$ is said to be convergent to a subset $A$ of $\mathcal{X}$ if:

(i) each point a in $A$ is the limit of a convergent sequence $\left\{a_{n}\right\}$, where $a_{n}$ is in $A_{n}$ for $n \in \mathbb{N}$,

(ii) for arbitrary $\varepsilon>0$, there exists an integer $m$ such that $A_{n} \subseteq A_{\varepsilon}$ for $n>m$, where $A_{\varepsilon}$ denotes the set of all points $x$ in $\mathcal{X}$ for which there

2000 Mathematics Subject Classification. Primary: 47H10, 54H25.

Key words and phrases. Commuting and weakly commuting maps, weakly compatible maps, compatible and compatible maps of type (A), (B) and (C), subcompatible maps, $D$-maps, implicit relations, single and set-valued maps, common fixed point theorems. 
exists a point a in $A$, depending on $x$, such that $d(x, a)<\epsilon . A$ is then said to be the limit of the sequence $\left\{A_{n}\right\}$.

Lemma 1.1 ([3]). If $\left\{A_{n}\right\}$ and $\left\{B_{n}\right\}$ are sequences in $B(\mathcal{X})$ converging to $A$ and $B$ in $B(\mathcal{X})$, respectively, then the sequence $\left\{\delta\left(A_{n}, B_{n}\right)\right\}$ converges to $\delta(A, B)$.

Lemma $1.2([4])$. Let $\left\{A_{n}\right\}$ be a sequence in $B(\mathcal{X})$ and $y$ be a point in $\mathcal{X}$ such that $\delta\left(A_{n}, y\right) \rightarrow 0$. Then the sequence $\left\{A_{n}\right\}$ converges to the set $\{y\}$ in $B(\mathcal{X})$.

To generalize commuting and weakly commuting maps, Jungck [5] introduced the concept of compatible maps. When $f$ and $g$ are self-maps of a metric space $(\mathcal{X}, d)$, he defines $f$ and $g$ to be compatible if

$$
\lim _{n \rightarrow \infty} d\left(f g x_{n}, g f x_{n}\right)=0
$$

whenever $\left\{x_{n}\right\}$ is a sequence in $\mathcal{X}$ such that $\lim _{n \rightarrow \infty} f x_{n}=\lim _{n \rightarrow \infty} g x_{n}=t$ for some $t \in \mathcal{X}$.

Further, Jungck et al. [7] gave another generalization of weakly commuting maps by introducing compatible maps of type $(A) . f$ and $g$ above are compatible of type $(A)$ if they satisfy instead of $(1)$ the two equalities

$$
\lim _{n \rightarrow \infty} d\left(f g x_{n}, g^{2} x_{n}\right)=0 \text { and } \lim _{n \rightarrow \infty} d\left(g f x_{n}, f^{2} x_{n}\right)=0 .
$$

Extending type $(A)$ maps, Pathak and Khan [10] introduced the notion of compatible maps of type $(B) . f$ and $g$ are compatible of type $(B)$ if in lieu of (1) we have

$$
\lim _{n \rightarrow \infty} d\left(f g x_{n}, g^{2} x_{n}\right) \leq \frac{1}{2}\left[\lim _{n \rightarrow \infty} d\left(f g x_{n}, f t\right)+\lim _{n \rightarrow \infty} d\left(f t, f^{2} x_{n}\right)\right]
$$

and

$$
\lim _{n \rightarrow \infty} d\left(g f x_{n}, f^{2} x_{n}\right) \leq \frac{1}{2}\left[\lim _{n \rightarrow \infty} d\left(g f x_{n}, g t\right)+\lim _{n \rightarrow \infty} d\left(g t, g^{2} x_{n}\right)\right] .
$$

In their paper [9], Pathak et al. added another extension of compatible maps of type $(A)$ by giving the concept of compatible maps of type $(C) . f$ and $g$ above are compatible of type $(C)$ if they satisfy the two inequalities

$$
\begin{aligned}
\lim _{n \rightarrow \infty} d\left(f g x_{n}, g^{2} x_{n}\right) \leq \frac{1}{3} & {\left[\lim _{n \rightarrow \infty} d\left(f g x_{n}, f t\right)\right.} \\
& \left.+\lim _{n \rightarrow \infty} d\left(f t, f^{2} x_{n}\right)+\lim _{n \rightarrow \infty} d\left(f t, g^{2} x_{n}\right)\right]
\end{aligned}
$$

and

$$
\begin{aligned}
\lim _{n \rightarrow \infty} d\left(g f x_{n}, f^{2} x_{n}\right) \leq \frac{1}{3} & {\left[\lim _{n \rightarrow \infty} d\left(g f x_{n}, g t\right)\right.} \\
& \left.+\lim _{n \rightarrow \infty} d\left(g t, g^{2} x_{n}\right)+\lim _{n \rightarrow \infty} d\left(g t, f^{2} x_{n}\right)\right] .
\end{aligned}
$$


In 1996, Jungck [6] gave a generalization of the above concepts by introducing the notion of weakly compatible maps. $f$ and $g$ are weakly compatible if they commute at their coincidence points, i.e., if $f t=g t$ for some $t \in \mathcal{X}$, then $f g t=g f t$.

Afterwards, Jungck and Rhoades [8] extended the above notion to the setting of single and set-valued maps. $f: \mathcal{X} \rightarrow \mathcal{X}$ and $F: \mathcal{X} \rightarrow B(\mathcal{X})$ are subcompatible if

$$
\{t \in \mathcal{X} / F t=\{f t\}\} \subseteq\{t \in \mathcal{X} / F f t=f F t\} .
$$

Recently, Djoudi and Khemis [2] introduced the concept of $D$-maps as follows: $f$ and $F$ above are $D$-maps if there exists a sequence $\left\{x_{n}\right\}$ in $\mathcal{X}$ such that

$$
\lim _{n \rightarrow \infty} f x_{n}=t \quad \text { and } \quad \lim _{n \rightarrow \infty} F x_{n}=\{t\}
$$

for some $t \in \mathcal{X}$.

\section{Example 1.1.}

(1) Let $\mathcal{X}=[1, \infty)$ with the usual metric $d$. Define $f: \mathcal{X} \rightarrow \mathcal{X}$ and $F: \mathcal{X} \rightarrow B(\mathcal{X})$ as follows

$$
f x=x \quad \text { and } \quad F x=[1, x] \quad \text { for } \quad x \in \mathcal{X} .
$$

Let $x_{n}=1+\frac{1}{n}$ for $n \in \mathbb{N}^{*}=\{1,2, \ldots\}$. Then,

$$
\lim _{n \rightarrow \infty} f x_{n}=\lim _{n \rightarrow \infty} x_{n}=1 \quad \text { and } \quad \lim _{n \rightarrow \infty} F x_{n}=\lim _{n \rightarrow \infty}\left[1, x_{n}\right]=\{1\} .
$$

Therefore $f$ and $F$ are $D$-maps.

(2) Endow $\mathcal{X}=[1, \infty)$ with the usual metric $d$ and define

$$
f x=x+3 \quad \text { and } \quad F x=[1, x] \quad \text { for every } \quad x \in \mathcal{X} .
$$

Suppose there exists a sequence $\left\{x_{n}\right\}$ in $\mathcal{X}$ such that $f x_{n} \rightarrow t$ and $y_{n} \rightarrow t$ for some $t \in \mathcal{X}$, with $y_{n} \in F x_{n}=\left[1, x_{n}\right]$. Then, $\lim _{n \rightarrow \infty} x_{n}=$ $t-3$ and $1 \leq t \leq t-3$, which is impossible.

Let $\mathbb{R}_{+}$be the set of all non-negative real numbers and $\mathcal{G}$ be the set of all continuous functions $G: \mathbb{R}_{+}^{6} \rightarrow \mathbb{R}$ satisfying the conditions

$\left(G_{1}\right): G$ is nondecreasing in variables $t_{5}$ and $t_{6}$,

$\left(G_{2}\right)$ : there exists $\theta \in(1, \infty)$, such that for every $u, v \geq 0$ with

$\left(G_{a}\right): G(u, v, u, v, u+v, 0) \geq 0$ or

$\left(G_{b}\right): G(u, v, v, u, 0, u+v) \geq 0$

we have $u \geq \theta v$.

$\left(G_{3}\right): G(u, u, 0,0, u, u)<0 \forall u>0$.

In [1], Djoudi established and proved the next result.

Theorem 1.1. Let $\mathcal{A}, \mathcal{B}, \mathcal{S}$ and $\mathcal{T}$ be maps from a complete metric space $\mathcal{X}$ into itself having the following conditions 
(i) $\mathcal{A}, \mathcal{B}$ are surjective,

(ii) the pairs of maps $\mathcal{A}, \mathcal{S}$ as well as $\mathcal{B}, \mathcal{T}$ are weakly compatible,

(iii) the inequality

$$
\begin{aligned}
& G(d(\mathcal{A} x, \mathcal{B} y), d(\mathcal{S} x, \mathcal{T} y), d(\mathcal{A} x, \mathcal{S} x), \\
& \quad d(\mathcal{B} y, \mathcal{T} y), d(\mathcal{A} x, \mathcal{T} y), d(\mathcal{B} y, \mathcal{S} x)) \geq 0
\end{aligned}
$$

for all $x, y \in \mathcal{X}$, where $G \in \mathcal{G}$. Then $\mathcal{A}, \mathcal{B}, \mathcal{S}$ and $\mathcal{T}$ have a unique common fixed point.

Our aim here is to extend the above result to the setting of single and set-valued maps in a metric space by deleting some conditions required on $G$. Also, we give a generalization of our result.

\section{IMPLICIT RELATIONS}

Let $\mathbb{R}_{+}$and let $\Phi$ be the set of all continuous functions $\varphi: \mathbb{R}_{+}^{6} \rightarrow \mathbb{R}$ satisfying the conditions

$\left(\varphi_{1}\right)$ : for every $u, v \geq 0$ with

$\left(\varphi_{a}\right): \varphi(u, v, u, v, u+v, 0) \geq 0$ or

$\left(\varphi_{b}\right): \varphi(u, v, v, u, 0, u+v) \geq 0$ we have $u \geq v$.

$\left(\varphi_{2}\right): \varphi(u, u, 0,0, u, u)<0 \forall u>0$.

\section{Example 2.1.}

$$
\varphi\left(t_{1}, t_{2}, t_{3}, t_{4}, t_{5}, t_{6}\right)=t_{1}^{p}-t_{2}^{p}-\frac{\alpha t_{5}^{p-1} t_{6}+\beta t_{5} t_{6}^{p-1}}{1+\gamma t_{3}^{p}+\delta t_{4}^{p}},
$$

where $\alpha, \beta>0, \gamma, \delta \geq 0$ and $p$ is an integer such that $p \geq 2$.

$\left(\varphi_{1}\right)$ : For $u \geq 0$ and $v \geq 0$ we have

$$
\varphi(u, v, u, v, u+v, 0)=\varphi(u, v, v, u, 0, u+v)=u^{p}-v^{p} \geq 0,
$$

which implies that $u \geq v$.

$\left(\varphi_{2}\right): \varphi(u, u, 0,0, u, u)=-(\alpha+\beta) u^{p}<0 \forall u>0$.

\section{Example 2.2.}

$$
\varphi\left(t_{1}, t_{2}, t_{3}, t_{4}, t_{5}, t_{6}\right)=t_{1}^{p}-a t_{2}^{p}-b t_{3}^{p}-c t_{4}^{p}-d t_{5}^{p-1} t_{6}-e t_{5} t_{6}^{p-1},
$$

where $a \geq 1,0 \leq b, c<1, a+b+c \geq 1, a+d+e>1$ and $p$ is an integer such that $p \geq 2$.

$\left(\varphi_{1}\right):$ For $u \geq 0$ and $v \geq 0$ we have

$$
\varphi(u, v, u, v, u+v, 0)=u^{p}-a v^{p}-b u^{p}-c v^{p} \geq 0
$$

which implies that

$$
u \geq\left(\frac{a+c}{1-b}\right)^{\frac{1}{p}} v \geq v .
$$


Similarly, we have

$$
\varphi(u, v, v, u, 0, u+v)=u^{p}-a v^{p}-b v^{p}-c u^{p} \geq 0
$$

which implies that

$$
u \geq\left(\frac{a+b}{1-c}\right)^{\frac{1}{p}} v \geq v .
$$

$\left(\varphi_{2}\right): \varphi(u, u, 0,0, u, u)=u^{p}(1-a-d-e)<0 \forall u>0$.

\section{Example 2.3.}

$$
\varphi\left(t_{1}, t_{2}, t_{3}, t_{4}, t_{5}, t_{6}\right)=\min \left\{t_{1}, t_{3}, t_{4}\right\}-k t_{1},
$$

where $k>1$.

$\left(\varphi_{1}\right)$ : Let $u \geq 0$ and $v \geq 0$. Suppose that $u<v$. Then

$$
\begin{aligned}
\varphi(u, v, u, v, u+v, 0) & =\varphi(u, v, v, u, 0, u+v)= \\
& =\min \{u, v\}-k u=u-k u \geq 0
\end{aligned}
$$

which implies that $u \geq k u>u$ which is a contradiction. Then $u \geq v$.

$\left(\varphi_{2}\right): \varphi(u, u, 0,0, u, u)=\min \{u, 0\}-k u=-k u<0, \forall u>0$.

\section{Example 2.4.}

$$
\varphi\left(t_{1}, t_{2}, t_{3}, t_{4}, t_{5}, t_{6}\right)=\min \left\{t_{1}^{2}, t_{3} t_{4}\right\}-\alpha t_{5} t_{6}-\beta t_{1}^{2},
$$

where $\alpha \geq 0$ and $\beta>1$.

$\left(\varphi_{1}\right)$ : Let $u \geq 0$ and $v \geq 0$. Suppose that $u<v$. Then

$$
\begin{aligned}
\varphi(u, v, u, v, u+v, 0) & =\varphi(u, v, v, u, 0, u+v)= \\
& =\min \left\{u^{2}, u v\right\}-\beta u^{2}=u^{2}-\beta u^{2} \geq 0
\end{aligned}
$$

which implies that $u^{2} \geq \beta u^{2}>u^{2}$, which is a contradiction. Then $u \geq v$.

$\left(\varphi_{2}\right): \varphi(u, u, 0,0, u, u)=\min \left\{u^{2}, 0\right\}-\alpha u^{2}-\beta u^{2}=-(\alpha+\beta) u^{2}<0$, $\forall u>0$.

\section{MAin RESUlts}

Theorem 3.1. Let $f, g$ be self-maps of a metric space $(\mathcal{X}, d)$ and let $F, G$ : $\mathcal{X} \rightarrow B(\mathcal{X})$ be two set-valued maps satisfying the conditions

(1) $f$ and $g$ are surjective,

(2) $\varphi(d(f x, g y), \delta(F x, G y), \delta(f x, F x), \delta(g y, G y), \delta(f x, G y), \delta(g y, F x)) \geq$ 0 for all $x, y$ in $\mathcal{X}$, where $\varphi \in \Phi$.

If either

(3) $f$ and $F$ are subcompatible D-maps; $g$ and $G$ are subcompatible, or

$\left(3^{\prime}\right) g$ and $G$ are subcompatible D-maps; $f$ and $F$ are subcompatible, 
then $f, g, F$ and $G$ have a unique common fixed point $t \in \mathcal{X}$ such that

$$
F t=G t=\{t\}=\{f t\}=\{g t\} .
$$

Proof. Suppose that $F$ and $f$ are $D$-maps, then, there exists a sequence $\left\{x_{n}\right\}$ in $\mathcal{X}$ such that $\lim _{n \rightarrow \infty} f x_{n}=t$ and $\lim _{n \rightarrow \infty} F x_{n}=\{t\}$ for some $t \in \mathcal{X}$. Since $f$ and $g$ are surjective, then, there exist two points $u$ and $v$ in $\mathcal{X}$ such that $t=f u=g v$. First, we show that $\{t\}=G v$. Indeed, by inequality (2) we get

$$
\begin{gathered}
\varphi\left(d\left(f x_{n}, g v\right), \delta\left(F x_{n}, G v\right), \delta\left(f x_{n}, F x_{n}\right), \delta(g v, G v),\right. \\
\left.\delta\left(f x_{n}, G v\right), \delta\left(g v, F x_{n}\right)\right) \geq 0 .
\end{gathered}
$$

Since $\varphi$ is continuous, using Lemma 1.1 we obtain at infinity

$$
\varphi(0, \delta(t, G v), 0, \delta(t, G v), \delta(t, G v), 0) \geq 0,
$$

thus, by $\left(\varphi_{a}\right)$ we have $G v=\{t\}$, i.e., $G v=\{t\}=\{g v\}$. Since $G$ and $g$ are subcompatible, then $G g v=g G v$ and hence $G G v=G g v=g G v=\{g g v\}$. We claim that $G g v=\{t\}$. Suppose not, then $\delta(t, G g v)>0$ and by (2) we get

$$
\begin{aligned}
\varphi\left(d\left(f x_{n}, g^{2} v\right), \delta\left(F x_{n}, G g v\right), \delta\left(f x_{n}, F x_{n}\right),\right. \\
\left.\delta\left(g^{2} v, G g v\right), \delta\left(f x_{n}, G g v\right), \delta\left(g^{2} v, F x_{n}\right)\right) \geq 0 .
\end{aligned}
$$

Since $\varphi$ is continuous, using lemma 1.1 we obtain at infinity

$$
\begin{aligned}
0 & \leq \varphi\left(d\left(t, g^{2} v\right), \delta(t, G g v), 0,0, \delta(t, G g v), \delta\left(g^{2} v, t\right)\right) \\
& =\varphi(\delta(t, G g v), \delta(t, G g v), 0,0, \delta(t, G g v), \delta(G g v, t))
\end{aligned}
$$

contradicts $\left(\varphi_{2}\right)$, then $G g v=\{t\}=\{g v\}=\{g g v\}$. , by inequality (2) we have

$$
\begin{aligned}
0 & \leq \varphi(d(f u, g v), \delta(F u, G v), \delta(f u, F u), \delta(g v, G v), \delta(f u, G v), \delta(g v, F u)) \\
& =\varphi(0, \delta(F u, t), \delta(t, F u), 0,0, \delta(t, F u))
\end{aligned}
$$

which by $\left(\varphi_{b}\right)$ implies that $F u=\{t\}=\{f u\}$. Since $F$ and $f$ are subcompatible, then $F f u=f F u$ and hence $F F u=F f u=f F u=\{f f u\}$. If $\delta(F f u, t)>0$, then by inequality (2) we have

$$
\begin{aligned}
0 & \leq \varphi\left(d\left(f^{2} u, g v\right), \delta(F f u, G v), \delta\left(f^{2} u, F f u\right),\right. \\
& \left.\delta(g v, G v), \delta\left(f^{2} u, G v\right), \delta(g v, F f u)\right)= \\
& =\varphi(\delta(F f u, t), \delta(F f u, t), 0,0, \delta(F f u, t), \delta(t, F f u))
\end{aligned}
$$

contradicts $\left(\varphi_{2}\right)$. Hence $F f u=\{t\}=\{f u\}=\{f f u\}$. Therefore $t=f u=$ $g v$ is a common fixed point of both $f, g, F$ and $G$.

Similarly, we can obtain this conclusion by using $\left(3^{\prime}\right)$ in lieu of $(3)$. 
Now, suppose that $f, g, F$ and $G$ have two common fixed points $t$ and $t^{\prime}$ such that $t^{\prime} \neq t$. Then inequality (2) gives

$$
\begin{aligned}
\varphi\left(d\left(f t, g t^{\prime}\right), \delta\left(F t, G t^{\prime}\right), \delta(f t, F t), \delta\left(g t^{\prime}, G t^{\prime}\right), \delta\left(f t, G t^{\prime}\right), \delta\left(g t^{\prime}, F t\right)\right) & = \\
= & \varphi\left(d\left(t, t^{\prime}\right), d\left(t, t^{\prime}\right), 0,0, d\left(t, t^{\prime}\right), d\left(t^{\prime}, t\right)\right) \geq 0
\end{aligned}
$$

contradicts $\left(\varphi_{2}\right)$. Therefore $t^{\prime}=t$.

If we let in the above theorem, $F=G$ and $f=g$ then we get the following result.

Corollary 3.1. Let $(\mathcal{X}, d)$ be a metric space and let $f: \mathcal{X} \rightarrow \mathcal{X}, F: \mathcal{X} \rightarrow$ $B(\mathcal{X})$ be a single and a set-valued map, respectively such that

(i) $f$ is surjective,

(ii) $\varphi(d(f x, f y), \delta(F x, F y), \delta(f x, F x), \delta(f y, F y)$, $\delta(f x, F y), \delta(f y, F x)) \geq 0$

for all $x, y$ in $\mathcal{X}$, where $\varphi \in \Phi$. If $f$ and $F$ are subcompatible $D$ maps, then, $f$ and $F$ have a unique common fixed point $t \in \mathcal{X}$ such that

$$
F t=\{t\}=\{f t\} .
$$

Now, if we put $f=g$ then we get the next corollary.

Corollary 3.2. Let $f$ be a self-map of a metric space $(\mathcal{X}, d)$ and let $F, G$ : $\mathcal{X} \rightarrow B(\mathcal{X})$ be two set-valued maps satisfying the conditions

(i) $f$ is surjective,

(ii) $\varphi(d(f x, f y), \delta(F x, G y), \delta(f x, F x), \delta(f y, G y)$, $\delta(f x, G y), \delta(f y, F x)) \geq 0$

for all $x, y$ in $\mathcal{X}$, where $\varphi \in \Phi$.

If either

(iii) $f$ and $F$ are subcompatible $D$-maps; $f$ and $G$ are subcompatible, or (iii $)^{\prime} f$ and $G$ are subcompatible D-maps; $f$ and $F$ are subcompatible.

Then, $f, F$ and $G$ have a unique common fixed point $t \in \mathcal{X}$ such that

$$
F t=G t=\{f t\}=\{t\} .
$$

Corollary 3.3. If in Theorem 3.1 we have instead of (2) the inequality

$$
\begin{aligned}
& d^{p}(f x, g y) \geq \delta^{p}(F x, G y)+ \\
& \quad+\frac{\alpha \delta^{p-1}(f x, G y) \delta(g y, F x)+\beta \delta(f x, G y) \delta^{p-1}(g y, F x)}{1+\gamma \delta^{p}(f x, F x)+\delta \delta^{p}(g y, G y)}
\end{aligned}
$$

for all $x, y$ in $\mathcal{X}$, where $\alpha, \beta>0, \gamma, \delta \geq 0$ and $p$ is an integer such that $p \geq 2$. Then, $f, g, F$ and $G$ have a unique common fixed point $t \in \mathcal{X}$. 
Proof. Take a function $\varphi$ as in Example 2.1, then

$$
\begin{gathered}
\varphi(d(f x, g y), \delta(F x, G y), \delta(f x, F x), \delta(g y, G y), \delta(f x, G y), \delta(g y, F x))= \\
=d^{p}(f x, g y)-\delta^{p}(F x, G y)- \\
-\frac{\alpha \delta^{p-1}(f x, G y) \delta(g y, F x)+\beta \delta(f x, G y) \delta^{p-1}(g y, F x)}{1+\gamma \delta^{p}(f x, F x)+\delta \delta^{p}(g y, G y)} \geq 0,
\end{gathered}
$$

which implies that

$$
\begin{aligned}
& d^{p}(f x, g y) \geq \delta^{p}(F x, G y)+ \\
& \qquad \frac{\alpha \delta^{p-1}(f x, G y) \delta(g y, F x)+\beta \delta(f x, G y) \delta^{p-1}(g y, F x)}{1+\gamma \delta^{p}(f x, F x)+\delta \delta^{p}(g y, G y)}
\end{aligned}
$$

for all $x, y$ in $\mathcal{X}$, where $\alpha, \beta>0, \gamma, \delta \geq 0$ and $p$ is an integer such that $p \geq 2$. Conclude by using Theorem 3.1.

Remark. As in Corollary 3.3 we can get other corollaries using Examples 2.2-2.4.

Corollary 3.4. Let $f, g, F$ and $G$ be maps satisfying (1), (3) and ( $\left.3^{\prime}\right)$ of Theorem 3.1. Suppose that for all $x, y \in \mathcal{X}$ we have the inequality $d^{p}(f x, g y) \geq \delta^{p}(F x, G y)+\delta^{p-1}(f x, G y) \delta(g y, F x)+\delta(f x, G y) \delta^{p-1}(g y, F x)$ where $p$ is an integer such that $p \geq 2$. Then, $f, g, F$ and $G$ have a unique common fixed point $t \in \mathcal{X}$.

Proof. Take a function $\varphi$ as in Example 2.1 with $\alpha=\beta=1$ and $\gamma=\delta=0$. Observe by condition (2)

$$
\begin{gathered}
\varphi(d(f x, g y), \delta(F x, G y), \delta(f x, F x), \delta(g y, G y), \delta(f x, G y), \delta(g y, F x))= \\
=d^{p}(f x, g y)-\delta^{p}(F x, G y)-\delta^{p-1}(f x, G y) \delta(g y, F x)- \\
\delta(f x, G y) \delta^{p-1}(g y, F x) \geq 0 .
\end{gathered}
$$

Conclude by using Theorem 3.1.

Remark. We can get other results if we let in the corollaries $f=g$ and also $f=g$ and $F=G$.

Now, we give a generalization of Theorem 3.1.

Theorem 3.2. Let $f, g$ be self-maps of a metric space $(\mathcal{X}, d)$ and $F_{n}: \mathcal{X} \rightarrow$ $B(\mathcal{X}), n \in \mathbb{N}^{*}=\{1,2, \ldots\}$ be set-valued maps with

(i) $f$ and $g$ are surjective,

(ii) the inequality

$$
\begin{gathered}
\varphi\left(d(f x, g y), \delta\left(F_{n} x, F_{n+1} y\right), \delta\left(f x, F_{n} x\right), \delta\left(g y, F_{n+1} y\right),\right. \\
\left.\delta\left(f x, F_{n+1} y\right), \delta\left(g y, F_{n} x\right)\right) \geq 0
\end{gathered}
$$

holds for all $x, y$ in $\mathcal{X}$, where $\varphi \in \Phi$. If either 
(iii) $f$ and $\left\{F_{n}\right\}_{n \in \mathbb{N}^{*}}$ are subcompatible D-maps; $g$ and $\left\{F_{n+1}\right\}_{n \in \mathbb{N}^{*}}$ are subcompatible, or

(iv) $g$ and $\left\{F_{n+1}\right\}_{n \in \mathbb{N}^{*}}$ are subcompatible D-maps; $f$ and $\left\{F_{n}\right\}_{n \in \mathbb{N}^{*}}$ are subcompatible.

Then, there is a unique common fixed point $t \in \mathcal{X}$ such that

$$
F_{n} t=\{t\}=\{f t\}=\{g t\}, \quad n \in \mathbb{N}^{*} .
$$

Proof. Letting $n=1$, we get the hypotheses of Theorem 3.1 for the maps $f, g, F_{1}$ and $F_{2}$ with the unique common fixed point $t$. Now, $t$ is a unique common fixed point of $f, g, F_{1}$ and of $f, g, F_{2}$. Otherwise, if $t^{\prime}$ is a second distinct fixed point of $f, g$ and $F_{1}$, then by inequality $(i i)$, we get

$$
\begin{aligned}
\varphi\left(d\left(f t^{\prime}, g t\right), \delta\left(F_{1} t^{\prime}, F_{2} t\right)\right. & , \delta\left(f t^{\prime}, F_{1} t^{\prime}\right), \delta\left(g t, F_{2} t\right), \delta\left(f t^{\prime}, F_{2} t\right), \\
\left.\delta\left(g t, F_{1} t^{\prime}\right)\right) & =\varphi\left(d\left(t^{\prime}, t\right), d\left(t^{\prime}, t\right), 0,0, d\left(t^{\prime}, t\right), d\left(t, t^{\prime}\right)\right) \geq 0
\end{aligned}
$$

which contradicts $\left(\varphi_{2}\right)$ hence $t^{\prime}=t$.

By the same method, we prove that $t$ is the unique common fixed point of the maps $f, g$ and $F_{2}$.

Now, by letting $n=2$, we get the hypotheses of Theorem 3.1 for the maps $f, g, F_{2}$ and $F_{3}$ and consequently they have a unique common fixed point $t^{\prime}$. Analogously, $t^{\prime}$ is the unique common fixed point of $f, g, F_{2}$ and of $f, g, F_{3}$. Thus $t^{\prime}=t$. Continuing in this way, we clearly see that $t$ is the required point.

\section{REFERENCES}

[1] A. Djoudi, General fixed point theorems for weakly compatible maps, Demonstratio Math, 38 (2005), No. 1, 197-205.

[2] A. Djoudi, R. Khemis, Fixed points for set and single valued maps without continuity, Demonstratio Math, 38 (2005), No. 3, 739-751.

[3] B. Fisher, Common fixed points of mappings and set-valued mappings, Rostock. Math. Kolloq, No. 18 (1981), 69-77.

[4] B. Fisher, S. Sessa, Two common fixed point theorems for weakly commuting mappings, Period. Math. Hungar, 20 (1989), No. 3, 207-218.

[5] G. Jungck, Compatible mappings and common fixed points, Internat. J. Math. \& Math. Sci., 9 (1986), No. 4, 771-779.

[6] G. Jungck, Common fixed points for noncontinuous nonself maps on nonmetric spaces, Far East J. Math. Sci., 4 (1996), No. 2, 199-215.

[7] G. Jungck, P.P. Murthy, Y.J. Cho, Compatible mappings of type (A) and common fixed points, Math. Japon., 38 (1993), No. 2, 381-390.

[8] G. Jungck, B.E. Rhoades, Fixed points for set valued functions without continuity, Indian J. Pure Appl. Math., 29 (1998), No. 3, 227-238. 
[9] H.K. Pathak, Y.J. Cho, S.M. Kang, B. Madharia, Compatible mappings of type $(C)$ and common fixed point theorems of Greguš type, Demonstratio Math., 31 (1998), No. 3, 499-518.

[10] H.K. Pathak, M.S. Khan, Compatible mappings of type $(B)$ and common fixed point theorems of Greguš type, Czechoslovak Math. J., 45(120) (1995), No. 4, 685-698.

H. BOUHADJERA

LABoratoire de Mathématiques AppliquéES

Université BADJi MOKHTAR

B.P. 12,23000, ANNABA

Algerie

E-mail address: b_hakima2000@yahoo.fr

\author{
A. Djoudi \\ Laboratoire de Mathématiques Appliquées \\ UNIVERSITÉ BADJI MOKHTAR \\ B.P. 12, 23000, ANNABA \\ AlGERIE \\ E-mail address: adjoudi@yahoo.com
}

BRIAN FISHER

Department of Mathematics

UNIVERSITY OF LEICESTER

LEICESTER, LE1 7RH,

U.K.

E-mail address: fbr@le.ac.uk 